\title{
A Hilbert-Type Integral Inequality with the Homogeneous Kernel of Degree -3 and a Best Constant Factor
}

\author{
${ }^{1}$ XIE Zitian and ${ }^{2}$ ZENG Zheng
}

${ }^{1}$ Dept. of Math. Zhaoqing University, Zhaoqing, Guangdong, 526061, P. R. China, ${ }^{2}$ Shaoguan University, Shaoguan, Guangdong, 512005 P. R. China.

Keywords. Hilbert-type integral inequality; weight function; Holder's inequality; equivalent form

Abstract. By establishing the weight function, we present a new Hilbert-type inequality with the integral in whole plane and with a best constant factor,and its kernel is a homogeneous form of degree-3, and also we put forward its equivalent form.

\section{Introduction}

If $f(x), g(x) \geq 0$, such that $0<\int_{0}^{\infty} f^{2}(x) \mathrm{d} x<\infty$ and $0<\int_{0}^{\infty} g^{2}(x) \mathrm{d} x<\infty$ then

$$
\int_{0}^{\infty} \int_{0}^{\infty} \frac{f(x) g(y)}{x+y} \mathrm{~d} x \mathrm{~d} y<\pi\left\{\int_{0}^{\infty} f(x) d x \int_{0}^{\infty} g(x) \mathrm{d} x\right\}^{1 / 2}
$$

where the constant factor $\pi$ is the best possible. Inequality (1.1) is well-known as Hilbert's integral inequality, which has been extended by Hardy-Riesz as [2]:

If $p>1,1 / p+1 / q=1, f(x), g(x) \geq 0$, such that

$$
0<\int_{0}^{\infty} f^{p}(x) \mathrm{d} x<\infty
$$
and $0<\int_{0}^{\infty} g^{q}(x) \mathrm{d} x<\infty$ then we have the following Hardy-Hilbert's integral inequality:

$$
\int_{0}^{\infty} \int_{0}^{\infty} \frac{f(x) g(y)}{x+y} \mathrm{~d} x \mathrm{~d} y<\frac{\pi}{\sin (\pi / p)}\left(\int_{0}^{\infty} f^{p}(x) \mathrm{d} x\right)^{1 / p}\left(\int_{0}^{\infty} g^{q}(x) \mathrm{d} x\right)^{1 / q} .
$$

Where the constant factor $\frac{\pi}{\sin (\pi / p)}$ also is the best possible.

In recent years, by introducing some parameters and estimating the way of weight function, inequalities (1.1) and (1.2) have many generalizations and variants. (1.1) has been strengthened by Yang and others. (including double series inequalities )[3-15].

In this paper, we give a new Hilbert-type inequality with the integral in whole plane.

In the following, we always suppose that: $\mathrm{p}>1,1 / \mathrm{p}+1 / \mathrm{q}=1, \mathrm{p} \neq 1, \mathrm{a}<1$.

\section{Some lemmas}

Lemma 2.1 Define the weight functions as follow:

$$
\begin{aligned}
& w(x):=\int_{-\infty}^{\infty} \frac{|x||y| d y}{(|x|+|y|)\left(y^{2}+2 a x y+x^{2}\right)}, w(y):=\int_{-\infty}^{\infty} \frac{y^{2} d x}{(|x|+|y|)\left(y^{2}+2 a x y+x^{2}\right)} . \\
& w(x)=w(y)=\frac{\pi}{2 \sqrt{1-a^{2}}} .
\end{aligned}
$$

Proof. We only prove that $w(x)=\frac{\pi}{2 \sqrt{1-a^{2}}}$ for $\mathrm{x} \in(-\infty, 0)$.

$w(x):=\int_{-\infty}^{0} \frac{|x||y| d y}{(|x|+|y|)\left(y^{2}+2 a x y+x^{2}\right)}+\int_{0}^{\infty} \frac{|x||y| d y}{(|x|+|y|)\left(y^{2}+2 a x y+x^{2}\right)}=w_{1}+w_{2}$. 
Setting $\mathrm{y}=\mathrm{tx}$, then

$$
\begin{aligned}
& w_{1}=\int_{-\infty}^{0} \frac{(-x)(-y) d y}{(-x-y)\left(y^{2}+2 a x y+x^{2}\right)}=\int_{0}^{\infty} \frac{t d t}{(t+1)\left(t^{2}+2 a t+1\right)}=\frac{1}{2(a-1)} \int_{0}^{\infty}\left(\frac{1}{t+1}-\frac{t+1}{t^{2}+2 a t+1}\right) d t \\
& =\frac{1}{2(a-1)}\left[\left.\ln \frac{t+1}{t^{2}+2 a t+1}\right|_{0} ^{\infty}-(1-a) \int_{0}^{\infty} \frac{d t}{t^{2}+2 a t+1}\right]=\frac{1}{2} \int_{0}^{\infty} \frac{d t}{t^{2}+2 a t+1}=\frac{1}{2 \sqrt{1-a^{2}}}\left(\frac{\pi}{2}-\arctan \frac{a}{\sqrt{1-a^{2}}}\right) .
\end{aligned}
$$

Setting $\mathrm{y}=-\mathrm{tx}$, then

$$
w_{2}=\int_{0}^{\infty} \frac{(-x) y d y}{(-x+y)\left(y^{2}+2 a x y+x^{2}\right)}=\int_{0}^{\infty} \frac{t d t}{(t+1)\left(t^{2}-2 a t+1\right)}=\frac{1}{2 \sqrt{1-a^{2}}}\left(\frac{\pi}{2}+\arctan \frac{a}{\sqrt{1-a^{2}}}\right)
$$

and $w(x)=\frac{\pi}{2 \sqrt{1-a^{2}}}$.

Easily if $\mathrm{x} \in(-\infty, 0)$, setting $\mathrm{x}=\mathrm{y} / \mathrm{t}$, we have

$$
\begin{aligned}
& w(y)=\int_{-\infty}^{\infty} \frac{y^{2} d x}{(|x|+|y|)\left(y^{2}+2 a x y+x^{2}\right)}=\int_{-\infty}^{0} \frac{y^{2} d x}{(|x|+|y|)\left(y^{2}+2 a x y+x^{2}\right)}+\int_{0}^{\infty} \frac{y^{2} d x}{(|x|+|y|)\left(y^{2}+2 a x y+x^{2}\right)} \\
& :=w_{1}+w_{2} \text {. And } \\
& w_{1}=\int_{-\infty}^{0} \frac{y^{2} d x}{(-x-y)\left(y^{2}+2 a x y+x^{2}\right)}=\int_{0}^{\infty} \frac{y^{2} d x}{(x+y)\left(y^{2}+2 a x y+x^{2}\right)}=\int_{0}^{\infty} \frac{y^{2} d\left(\frac{y}{t}\right)}{\left(\left(\frac{y}{t}\right)+y\right)\left(y^{2}+2 a\left(\frac{y}{t}\right) y+\left(\frac{y}{t}\right)^{2}\right)}
\end{aligned}
$$$$
=\int_{0}^{\infty} \frac{t d t}{(t+1)\left(t^{2}+2 a t+1\right)}=w_{1} .
$$

Similarly, $\mathrm{w}_{2}(\mathrm{x})=\mathrm{w}_{2}$, and $w(x)=w(y)=\frac{\pi}{2 \sqrt{1-a^{2}}}$

The lemma is proved.

Lemma 2.2 For $\frac{q}{2}>\varepsilon>$, define both functions, $f$ and $g$ as follow:

$$
f(x)=\left\{\begin{array}{ll}
x^{-2 \varepsilon / p}, & \text { if } x \in(1, \infty), \\
0, & \text { if } x \in[-1,1], \\
(-x)^{-2 \varepsilon / p}, & \text { if } x \in(-\infty, 1) ;
\end{array} \quad \text { and } g(x)= \begin{cases}x^{1-2 \varepsilon / q}, & \text { if } x \in(1, \infty), \\
0, & \text { if } x \in[-1,1], \\
(-x)^{1-2 \varepsilon / q}, & \text { if } x \in(-\infty, 1)\end{cases}\right.
$$

Then

$$
\begin{aligned}
& I(\varepsilon):=\varepsilon\left\{\int_{-\infty}^{\infty}|x|^{-1} f^{p}(x) d x\right\}^{1 / p}\left\{\int_{-\infty}^{\infty}|x|^{-q-1} g^{q}(x) d x\right\}^{1 / p}=1 \\
& \tilde{I}(\varepsilon):=2 \varepsilon \int_{-\infty}^{\infty} \int_{-\infty}^{\infty} \frac{f(x) g(y)}{(|x|+|y|)\left(y^{2}+2 a x y+x^{2}\right)} d x d y=\frac{\pi}{2 \sqrt{1-a^{2}}}+o(1),\left(\text { for } \varepsilon \rightarrow 0^{+}\right)
\end{aligned}
$$

Proof Easily,

$$
I(\varepsilon):=2 \varepsilon\left\{\int_{1}^{\infty} x^{-1} x^{-2 \varepsilon} d x\right\}^{1 / p}\left\{\int_{0}^{1} x^{-1} x^{-2 \varepsilon} d x\right\}^{1 / p}=1
$$


Let $y=-Y$, using $(f-x)=f(x), g(-x)=g(x)$ and

$$
\begin{aligned}
& f(-x) \int_{-\infty}^{\infty} \frac{g(y) d y}{(|x|+|y|)\left(y^{2}-2 a x y+x^{2}\right)}=f(x) \int_{-\infty}^{\infty} g(Y) \frac{g(Y) d Y}{(|x|+|Y|)\left(Y^{2}+2 a x Y+x^{2}\right)} \\
& f(x) \int_{-\infty}^{\infty} \frac{g(y) d y}{(|x|+|y|)\left(y^{2}+2 a x y+x^{2}\right)} \quad \text { is an even function,then }
\end{aligned}
$$

we have that

$$
\begin{aligned}
\tilde{I}(\varepsilon) & =2 \varepsilon \int_{0}^{\infty} f(x)\left(\int_{-\infty}^{\infty} \frac{g(y) d y}{(|x|+|y|)\left(y^{2}+2 a x y+x^{2}\right)}\right) d x \\
& =2 \varepsilon\left[\int_{1}^{\infty} x^{-2 \varepsilon / p}\left(\int_{-\infty}^{-1} \frac{(-y)^{1-\frac{2 \varepsilon}{q}} d y}{(x-y)\left(y^{2}+2 a x y+x^{2}\right)}\right) d x+\int_{1}^{\infty} x^{-2 \varepsilon / p}\left(\int_{1}^{\infty} \frac{y^{1-\frac{2 \varepsilon}{q}} d y}{(x+y)\left(y^{2}+2 a x y+x^{2}\right)}\right) d x\right] \\
& =I_{1}+I_{2} .
\end{aligned}
$$

Setting $y=x u$ then

$$
\begin{aligned}
& I_{1}=2 \varepsilon \int_{1}^{\infty} x^{-2 \varepsilon / p}\left(\int_{1}^{\infty} \frac{y^{1-\frac{2 \varepsilon}{q}}}{(x+y)\left(y^{2}-2 a x y+x^{2}\right)} d y\right) d x=2 \varepsilon \int_{1}^{\infty} x^{-1-2 \varepsilon}\left(\int_{\frac{1}{x}}^{\infty} \frac{u^{1-\frac{2 \varepsilon}{q}}}{(u+1)\left(u^{2}-2 a u+1\right)} d u\right) d x \\
& =2 \varepsilon\left[\int_{1}^{\infty} x^{-1-2 \varepsilon}\left(\int_{1}^{\infty} \frac{u^{1-\frac{2 \varepsilon}{q}}}{(u+1)\left(u^{2}-2 a u+1\right)} d u\right) d x+\int_{1}^{\infty} x^{-1-2 \varepsilon}\left(\int_{\underline{x}}^{\infty} \frac{u^{1-\frac{2 \varepsilon}{q}}}{(u+1)\left(u^{2}-2 a u+1\right)} d u\right) d x\right] \\
& =\int_{1}^{\infty} \frac{u^{1-\frac{2 \varepsilon}{q}}}{(u+1)\left(u^{2}-2 a u+1\right)} d u+2 \varepsilon \int_{0}^{1} \frac{u^{1-\frac{2 \varepsilon}{q}}}{(u+1)\left(u^{2}-2 a u+1\right)}\left(\int_{1 / u}^{\infty} x^{-1-2 \varepsilon} d x\right) d u \\
& =\int_{1}^{\infty} \frac{u^{1-\frac{2 \varepsilon}{q}}}{(u+1)\left(u^{2}-2 a u+1\right)} d u+\int_{0}^{1} \frac{u^{1+\frac{2 \varepsilon}{p}}}{(u+1)\left(u^{2}-2 a u+1\right)} d u \\
& =\int_{0}^{\infty} \frac{u^{1-\frac{2 \varepsilon}{q}}}{(u+1)\left(u^{2}-2 a u+1\right)} d u+\int_{0}^{1}\left(u^{2 \varepsilon / p}-u^{-2 \varepsilon / q}\right) \frac{u}{(u+1)\left(u^{2}-2 a u+1\right)} d u \\
& =\int_{0}^{\infty} \frac{u^{1-\frac{2 \varepsilon}{q}}}{(u+1)\left(u^{2}-2 a u+1^{2}\right)} d u+\eta(\varepsilon) \\
& =\frac{1}{2 \sqrt{1-a^{2}}}\left(\frac{\pi}{2}+\arctan \frac{a}{\sqrt{1-a^{2}}}\right)+o(1)\left(\text { for } \varepsilon \rightarrow 0^{+}\right) \text {. }
\end{aligned}
$$

The reason for the last equation is that, because of $\exists \mathrm{M} \in \square_{+}$, such that

$$
\int_{0}^{\infty} \frac{u^{1-\frac{2 \varepsilon}{q}}}{(u+1)\left(u^{2}-2 a u+1\right)} d u<\int_{0}^{1} \frac{1}{(u+1)\left(u^{2}-2 a u+1\right)} d u+\int_{0}^{\infty} \frac{u}{(u+1)\left(u^{2}-2 a u+1\right)} d u<\mathrm{M}
$$

and by using the theorem of control convergence, we have the conclusion as follows:

$$
\lim _{\varepsilon \rightarrow 0^{+}} \int_{1}^{\infty} \frac{u^{1-\frac{2 \varepsilon}{q}}}{(u+1)\left(u^{2}-2 a u+1\right)} d u=\int_{1}^{\infty} \frac{u}{(u+1)\left(u^{2}-2 a u+1\right)} d u=\frac{1}{2 \sqrt{1-a^{2}}}\left(\frac{\pi}{2}+\arctan \frac{a}{\sqrt{1-a^{2}}}\right),
$$


and we have $I_{1} \rightarrow \frac{1}{2 \sqrt{1-a^{2}}}\left(\frac{\pi}{2}+\arctan \frac{a}{\sqrt{1-a^{2}}}\right)\left(\varepsilon \rightarrow 0^{+}\right)$

Similarly $I_{2} \rightarrow \frac{1}{2 \sqrt{1-a^{2}}}\left(\frac{\pi}{2}-\arctan \frac{a}{\sqrt{1-a^{2}}}\right)$ (for $\varepsilon \rightarrow 0^{+}$).

Lemma 2.3. If ${ }^{0<\int_{-\infty}^{\infty}|x|^{-1} f^{p}(x) \mathrm{d} x<\infty}$ we have

$$
J:=\int_{-\infty}^{\infty}|y|^{2 p-1}\left(\int_{-\infty}^{\infty} \frac{f(x)}{(|x|+|y|)\left(y^{2}+2 a x y+x^{2}\right)} d x\right)^{p} d y \leq\left(\frac{\pi}{2 \sqrt{1-a^{2}}}\right)^{p} \int_{-\infty}^{\infty}|x|^{-1} f^{p}(x) \mathrm{d} x
$$

Proof By lemma 2.2,we find

$$
\begin{aligned}
& \left(\int_{-\infty}^{\infty} \frac{f(x)}{(|x|+|y|)\left(y^{2}+2 a x y+x^{2}\right)} d x\right)^{p} \\
& =\left(\int_{-\infty}^{\infty} \frac{1}{(x|+| y \mid)\left(y^{2}+2 a x y+x^{2}\right)}|y|^{1 / p} f(x)|y|^{-1 / p} d x\right)^{p} \\
& \leq \int_{-\infty}^{\infty} \frac{|y|}{(|x|+|y|)\left(y^{2}+2 a x y+x^{2}\right)} f^{p}(x) d x\left(\int_{-\infty}^{\infty} \frac{|y|^{1-q}}{(|x|+|y|)\left(y^{2}+2 a x y+x^{2}\right)} d x\right)^{p-1} \\
& =\left(\frac{\pi}{2 \sqrt{1-a^{2}}}\right)^{p-1}|y|^{-2 p+1} \int_{-\infty}^{\infty} \frac{|y|}{(|x|+|y|)\left(y^{2}+2 a x y+x^{2}\right)} f^{p}(x) d x, \\
& J \leq\left(\frac{\pi}{2 \sqrt{1-a^{2}}}\right)^{p-1} \int_{-\infty}^{\infty}\left[\int_{-\infty}^{\infty} \frac{|y|}{(|x|+|y|)\left(y^{2}+2 a x y+x^{2}\right)} f^{p}(x) d x\right] d y \\
& =\left(\frac{\pi}{2 \sqrt{1-a^{2}}}\right)^{p-1} \int_{-\infty}^{\infty}\left[\int_{-\infty}^{\infty} \frac{|y|}{(|x|+|y|)\left(y^{2}+2 a x y+x^{2}\right)} d y\right] f^{p}(x) d x \\
& =\left(\frac{\pi}{2 \sqrt{1-a^{2}}}\right)^{p} \int_{-\infty}^{\infty}|x|^{-1} f^{p}(x) \mathrm{d} x \quad
\end{aligned}
$$

\section{Main results}

Theorem. If both functions, $\mathrm{f}(\mathrm{x})$ and $\mathrm{g}(\mathrm{x})$ are nonnegative measurable functions, and satisfy

$$
\begin{gathered}
0<\int_{-\infty}^{\infty}|x|^{p r-1} f^{p}(x) \mathrm{d} x<\infty \text {, and } 0<\int_{-\infty}^{\infty}|x|^{-q-1} g^{q}(x) \mathrm{d} x<\infty, \text { then } \\
I^{*}:=\int_{-\infty}^{\infty} \int_{-\infty}^{\infty} \frac{f(x) g(y)}{(|x|+|y|)\left(y^{2}+2 a x y+x^{2}\right)} d x d y \\
<\frac{\pi}{2 \sqrt{1-a^{2}}}\left(\int_{-\infty}^{\infty}|x|^{-1} f^{p}(x) \mathrm{d} x\right)^{1 / p}\left(\int_{-\infty}^{\infty}|x|^{-q-1} g^{q}(x) \mathrm{d} x\right)^{1 / q},
\end{gathered}
$$


and

$$
J=\int_{-\infty}^{\infty}|y|^{2 p-1}\left(\int_{-\infty}^{\infty} \frac{f(x)}{(|x|+|y|)\left(y^{2}+2 a x y+x^{2}\right)} d x\right)^{p} d y<\left(\frac{\pi}{2 \sqrt{1-a^{2}}}\right)^{p} \int_{-\infty}^{\infty}|x|^{-1} f^{p}(x) \mathrm{d} x
$$

Inequalities (3.1)and (3.2) are equivalent, and the constant factors in the two forms are all the best possible.

Proof If (2.5) takes the form of equality for some $y \in(-\infty, 0) \cup(0, \infty)$, then there exist constants $M$ and $N$, such that they are not all zero, and

$$
M|y| f^{p}(x)=N|y|^{-q-1} \text { a.e. in }(-\infty, \infty) \times(-\infty, \infty),
$$

Hence, there exists a constantC, such that

$$
M f^{p}(x)=N|y|^{-q}=C \quad \text { a.e. in }(-\infty, \infty) \times(-\infty, \infty) \text {. }
$$

We claim that $\mathrm{M}=0$. In fact, if $\mathrm{M} \neq 0$, then $|x|^{-1} f^{p}(x)=C /(M|x|)$ a.e. in $(-\infty, \infty)$ which contradicts the fact that $0<\int_{-\infty}^{\infty}|x|^{-1} f^{p}(x) \mathrm{d} x<\infty$.. In the same way, we claim that $\mathrm{N}=0$. This is too a contradiction and hence by (2.7), we have (3.2).

By Holder's inequality with weight and (3.2), we have,

$$
\begin{aligned}
I^{*} & =\int_{-\infty}^{\infty}\left[|y|^{1+1 / q} \int_{-\infty}^{\infty} \frac{f(x)}{(|x|+|y|)\left(y^{2}+2 a x y+x^{2}\right)} d x\right]\left[|y|^{-1-1 / q} g(y)\right] d y \\
& \leq(J)^{1 / p}\left(\int_{-\infty}^{\infty}|y|^{-q-1} g^{q}(y) \mathrm{d} y\right)^{1 / q}
\end{aligned}
$$

Using (3.2), we have (3.1).

Setting $g(y)=|y|^{2 p-1}\left(\int_{-\infty}^{\infty} \frac{f(x)}{(|x|+|y|)\left(y^{2}+2 a x y+x^{2}\right)} d x\right)^{p-1}$,then $J=\int_{-\infty}^{\infty}|y|^{-q-1} g^{q} y \mathrm{~d} y$ by (2.7)

we have $\mathrm{J}<\infty$.if $\mathrm{J}=0$ then (3.2) is proved; if $0<\mathrm{J}<\infty$, by (3.1), we obtain

$$
\begin{gathered}
0<\int_{-\infty}^{\infty}|y|^{-q-1} g^{q}(y) \mathrm{d} y=J=I^{*}<\frac{\pi}{2 \sqrt{1-a^{2}}}\left(\int_{-\infty}^{\infty}|x|^{-1} f^{p}(x) \mathrm{d} x\right)^{1 / p}\left(\int_{-\infty}^{\infty}|x|^{-q-1} g^{q}(x) \mathrm{d} x\right)^{1 / q} \\
\left(\int_{-\infty}^{\infty}|x|^{-q-1} g^{q}(x) \mathrm{d} x\right)^{1 / p}=J^{1 / p}<\frac{\pi}{2 \sqrt{1-a^{2}}}\left(\int_{-\infty}^{\infty}|x|^{-1} f^{p}(x) \mathrm{d} x\right)^{1 / p}
\end{gathered}
$$

Inequalities (3.1)and (3.2) are equivalent.

If the constant factor $\frac{\pi}{2 \sqrt{1-a^{2}}}$ in (3.1) is not the best possible, then there exists a positive $h$ (with $h<\frac{\pi}{2 \sqrt{1-a^{2}}}$ ), such that

$$
\int_{-\infty}^{\infty} \int_{-\infty}^{\infty} \frac{f(x) g(y)}{(|x|+|y|)\left(y^{2}+2 a x y+x^{2}\right)} d x d y<h\left(\int_{-\infty}^{\infty}|x|^{-1} f^{p}(x) \mathrm{d} x\right)^{1 / p}\left(\int_{-\infty}^{\infty}|x|^{-q-1} g^{q}(x) \mathrm{d} x\right)^{1 / q}
$$

For $>>$ 0,by (3.4), using lemma 2.3,we have

$$
\tilde{I}(\varepsilon)=k+o(1)<\varepsilon h\left(\int_{-\infty}^{\infty}|x|^{-1} f^{p}(x) \mathrm{d} x\right)^{1 / p}\left(\int_{-\infty}^{\infty}|x|^{-q-1} g^{q}(x) \mathrm{d} x\right)^{1 / q}=h
$$


Hence we find, $\frac{\pi}{2 \sqrt{1-a^{2}}}+o(1)<h$ for $\varepsilon 0^{+} \rightarrow$, it follows that $\frac{\pi}{2 \sqrt{1-a^{2}}} \leq h$ which contradicts the

fact that $h<\frac{\pi}{2 \sqrt{1-a^{2}}}$ Hence the constant $h$ in (3.1) is the best possible.As (3.1) and (3.2) are equivalent,if the constant factor in (3.2) is not the best possible, then by using (3.2), we can get a contradiction that the constant factor in (3.1) is not the best possible.

Thus we complete the prove of the theorem.

Remark For $\alpha=\frac{\pi}{4}, \beta=\frac{\pi}{3}$ in (3.1), we have the following particular result:

$\int_{-\infty}^{\infty} \int_{-\infty}^{\infty} \frac{f(x) g(y)}{(|x|+|y|)\left(y^{2}+2 x y \cos \alpha+x^{2}\right)} d x d y<\frac{\pi}{2 \sin \alpha}\left(\int_{-\infty}^{\infty}|x|^{-1} f^{p}(x) \mathrm{d} x\right)^{1 / p}\left(\int_{-\infty}^{\infty}|x|^{-q-1} g^{q}(x) \mathrm{d} x\right)^{1 / q}$.

Where the constant factor $\frac{\pi}{2 \sin \alpha}$ also is the best possible.

\section{References}

[1]Hardy G.H.,Littlewood J E.and Polya G, Inequalities, Cambridge University Press,Cambridge, 1952.

[2] Hardy G. H., Note on a theorem of Hilbert concerning series of positive terems, Proceedings London Math. Soc, 1925,23(2): Records of Proc. XLV-XLVI.

[3]Bicheng Yang, A new Hilbert-type integral inequality with a parameters, Journal of Henan University(NaturalScience), 2005,35(4): 4-8

[4] Zitian Xie and Zeng Zheng ,A Hilbert-type integral inequality whose kernel Is a homogeneous form of degree -3.Math.Anal.Appl.,2008,339;324-331

[5] Bicheng Yang, A new Hilbert-type integral inequality with some parameters,Journal of Jilin University(Science Edition), 2008,46(6) : 1085-1090.

[6]Xie Zitian, A New Hilbert-type integral inequality with the homogeneous kernel of real numberdegre, Journal of Jishou University(Naturnal Science Edition), 2011,32(4), 26-30

[7]Zitian Xie and Zheng Zeng,A Hilbert-type integral Iinequality with a non-homogeneous form and a best constant factor,Advances and applications in mathematical sciences, 2010, 3,(1),61-71

[8]Zheng Zeng and Zitian Xie, On a new Hilbert-type integral inequality with the the integral in whole plane,Journal of Inequalities and Applications , vol. 2010, Article ID 256796, 8 pages, 2010. doi:10.1155/2010/256796

[9]Zitian Xie,Bicheng Yang, Zheng Zeng,A New Hilbert-type integral inequality with the homogeneous kernel of real number-degree ,Journal of Jilin University(Science Edition), 2010,48(6)941-945.

[10]Zitian Xie and Benlu Fu, A new Hilbert-type integral inequality with the best constant factor, J. Wuhan Univ. (Nat.Sci.Ed) 2009,55(6):637-640.

[11]Xie Zitian,Zeng Zheng, A new half-discrete Hilbert-type inequality with the homogeneous kernel of degree, Journal of Jishou University(Naturnal Science Edition), 2012,33 (2) , 15-19. [12]Zitian Xie and Xingdong Liu, A new Hilbert-type integral inequality and its reverse, Journal of Henan University (Science Edition), 2009, 39(1): 10-13.

[13]Zitian Xie,Zheng Zeng,Qinghua Zhou ,A new Hilbert-type integral inequality with the homogeneous kernel of real number-degree and its equivalent inequality forms,Journal of Jilin University(Science Edition), 2012,50(4), 693-697.

[14]Xie Zitian,Liang Hongwei, A generalization of Hilbert-type inequality, Journal of Henan University (Natural Science), 2012, 42 (2) , 117-120.

[15]Zitian Xie, A Hilbert-type integral inequality with a mix homogeneous kernel of real numberdegree, Journal of South China Normal University (Natural Science Edition), 2012,44(3),36-39 